\title{
Defects in mTORC1 Network and mTORC1-STAT3 Pathway Crosstalk Contributes to Non-inflammatory Hepatocellular Carcinoma
}

\begin{abstract}
Ting Li ${ }^{* *}$, Guohong Zhang ${ }^{1,2,3}$, Linlin Wang ${ }^{4,5}$, Susu Li5, Xiaoping $X u^{1,3,6,7 *}$ and Yi Gao ${ }^{1,3,6,7 *}$
${ }^{1}$ Department of Hepatobiliary Surgery II, Zhujiang Hospital, Southern Medical University, Guangzhou, China, ${ }^{2}$ Department of Pathology, Shantou University Medical College, Shantou, China, ${ }^{3}$ Institute of Regenerative Medicine, Zhujiang Hospital, Southern Medical University, Guangzhou, China, ${ }^{4}$ Department of Oncology, Nanfang Hospital, Southern Medical University, Guangzhou, China, ${ }^{5}$ Department of Cell Biology, School of Basic Medical Sciences, Southern Medical University, Guangzhou, China, ${ }^{6}$ Artificial Organs and Tissue Engineering Centre of Guangdong Province, Guangzhou, China, ' State Key Laboratory of Organ Failure Research, Southern Medical University, Guangzhou, China
\end{abstract}

OPEN ACCESS

Edited by:

Zhe-Sheng Chen

St. John's University, United States

Reviewed by:

Xingxiang Pu,

Hunan Cancer Hospital, China

Xiaozhuo Liu,

University at Buffalo, United States

*Correspondence:

Ting $\mathrm{Li}$

373663456@qq.com

Xiaoping $X u$

xuxp7193@163.com

Yi Gao

gaoyi6146@163.com

Specialty section:

This article was submitted to Molecular and Cellular Oncology,

a section of the journal

Frontiers in Cell and Developmental

Biology

Received: 02 February 2020

Accepted: 16 March 2020

Published: 07 April 2020

Citation:

Li T, Zhang G, Wang L, Li S, Xu X and Gao Y (2020) Defects in mTORC1

Network and mTORC1-STAT3

Pathway Crosstalk Contributes to Non-inflammatory Hepatocellular

Carcinoma

Front. Cell Dev. Biol. 8:225 doi: 10.3389/fcell.2020.00225
Background and Aims: Mammalian target of rapamycin complex 1 (mTORC1) is frequently hyperactivated in hepatocellular carcinoma (HCC). Cases of HCC without inflammation and cirrhosis are not rarely seen in clinics. However, the molecular basis of non-inflammatory HCC remains unclear.

Methods: Spontaneous non-inflammatory HCC in mice was triggered by constitutive elevation of mTORC1 by liver-specific TSC1 knockout (LTSC1KO). A multi-omics approach was utilized on tumor tissues to better understand the molecular basis for the development of HCC in the LTSC1KO model.

Results: We showed that $L T S C 1 K O$ in mice triggered spontaneous non-inflammatory HCC, with molecular characteristics similar to those of diethylnitrosamine-mediated non-cirrhotic HCC. Mitochondrial and autophagy defects, as well as hepatic metabolic disorder were manifested in HCC development by LTSC1KO. mTORC1 activation on its own regulated an oncogenic network (DNA-damage-inducible transcript 4, nuclear protein 1, and fibroblast growth factor 21), and mTORC1-signal transducer and activator of transcription pathway crosstalk that altered specific metabolic pathways contributed to the development of non-inflammatory HCC.

Conclusion: Our findings reveal the mechanisms of mTORC1-driven non-inflammatory $\mathrm{HCC}$ and provide insight into further development of a protective strategy against noninflammatory HCC.

Keywords: carcinogenesis, mouse model, non-inflammation, gene network, metabolic disorder 


\section{INTRODUCTION}

Hepatocellular carcinoma (HCC) is the fifth most common cancer in men and the seventh in women, and is now the second most common cause of cancer-related death worldwide (Bosetti et al., 2014; Inarrairaegui et al., 2018). Patients with advanced HCC have limited treatment options, and chemotherapy provides minimal survival benefit. Lack of knowledge of the mechanisms of HCC is one of the important factors that limit the development of HCC treatment. Therefore, understanding the underlying mechanisms of HCC is still urgently needed to explore novel therapeutic options.

Hepatitis B or hepatitis $\mathrm{C}$ is the leading risk factor for HCC, and other risk factors include obesity, diabetes and related nonalcoholic fatty liver disease (NAFLD) (Yu and Yuan, 2004; Mittal and El-Serag, 2013). Chronic inflammation is closely associated with persistent hepatic injury and concurrent regeneration, leading to sequential development of fibrosis, cirrhosis, and eventually HCC (Bishayee, 2014). However, cases of HCC in the absence of inflammation and cirrhosis are not rare in clinics (Guzman et al., 2008; Mohamad et al., 2016). Most studies have focused on the mechanisms of chronic-inflammation-based HCC, while few studies have been conducted on the molecular basis of non-inflammatory HCC in animal models (Moriya et al., 2001). The mechanisms of HCC in the absence of inflammation are still undefined and need further investigation.

The mammalian target of rapamycin (mTOR) pathway is aberrantly up-regulated in up to $50 \%$ of HCCs (Bhat et al., 2013). The mTOR kinase nucleates two distinct protein complexes termed mTOR complex 1 (mTORC1) and complex 2 (mTORC2). The mTOR pathway regulates key cellular functions linked to the promotion of cell growth and metabolism (Guertin and Sabatini, 2007). Various signaling pathways upstream of mTORC1 stimulate its activity through inhibition of the tuberous sclerosis (TSC) 1-TSC2 complex. Disruption of this complex, through the loss of TSC1 or TSC2, results in ectopic activation of mTORC1 (Laplante and Sabatini, 2009). Dysfunction of hepatic TSC1, a suppressor of mTOR signaling, occurs in patients with hepatitis $B$, hepatitis $\mathrm{C}$ or insulin resistance, which is a risk factor for HCC development (Lee et al., 2008; Totoki et al., 2011; Yen et al., 2012; Ho et al., 2017). Menon et al. (2012) detected sporadic HCC development in a liver-specific TSC1 knockout (LTsc1KO) mouse model and proposed that the liver damaging, inflammatory cycles of necrosis and regeneration may contribute to the development of HCC in LTsc1KO mice. However, in this study, we did not find any obvious histological evidence of inflammation, necrosis and fibrosis in LTsc1KO HCC mice. These mice are a good model to investigate the underlying molecular mechanisms of HCC development in the absence of inflammation. We utilized a multiomics strategy including transcriptomics, cytokine proteomics, genomics and metabolomics, to determine the comprehensive molecular basis for without long-term hepatic inflammation, necrosis, or fibrosis.
Our findings demonstrate that inflammation and fibrosis are not prerequisites in HCC development triggered by TSC1 deficiency. Non-inflammatory HCC developed by LTsc1KO displayed molecular characteristics that were similar to those of diethylnitrosamine (DEN)-mediated non-cirrhotic HCC (Chen et al., 2015). Mitochondrial and autophagy defects, as well as hepatic steatosis were manifested in HCC. mTORC1 activation on its own regulated a gene network, including DNA-damage-inducible transcript 4 (Ddit4), nuclear protein 1 (Nurp1) and fibroblast growth factor 21 (FGF21), and mTORC1-signal transducer and activator of transcription pathway crosstalk that altered specific metabolic pathways contributed to the development of non-inflammatory HCC.

\section{MATERIALS AND METHODS}

\section{Generation of Mice}

Mice carrying the $T s c 1^{\mathrm{fl}}$ allele in the FVB background have been previous described (Yecies et al., 2011). LTsc1KO mice were generated by crossing $T S C 1^{\mathrm{fl} / f l}$ mice with Alb-Cre mice (C57BL/6J background) obtained from Jackson Laboratory (Farmington, CT, United States) (Kwiatkowski et al., 2002). The specificity of recombination was confirmed by PCR using primers flanking the floxed allele. The primer sequences are listed in Supplementary Table S1. All procedures involving mice was approved by the Southern Medical University Animal Care and Use Committee. Mice importing, transporting, housing, and breeding were conducted according to the recommendations of "The use of non-human primates in research.”

\section{Mouse Diets and Treatments}

The mice were housed in plastic cages at a controlled temperature of $22 \pm 1^{\circ} \mathrm{C}$ on a $12 \mathrm{~h}$ light/12 $\mathrm{h}$ dark cycle with lights on from 06:00 to 18:00 h. Standard rodent chow and water were provided ad libitum throughout the entire feeding period. All of the animal experiments were approved by the Animal Ethics Committee of Southern Medical University (approval number SYXK 2011-0074) and performed in accordance with animal ethics guidelines and approved protocols. Mice aged 5 months were subjected to normal saline (NS) or rapamycin gavage. For rapamycin treatment, mice were administered by oral gavage $5 \mathrm{mg} / \mathrm{kg} /$ day rapamycin until killing at 10 months of age, and the same volume of NS was administered to control mice. All mice were killed prior to their daily feeding.

\section{Statistical Analysis}

Continuous variables were compared using independent sample $t$-tests or one-way analysis of variance, with the data expressed as mean \pm SEM. Significant differences are indicated in the figure legends, unless otherwise indicated.

Other detailed experimental procedures are described in Supplementary Material. 


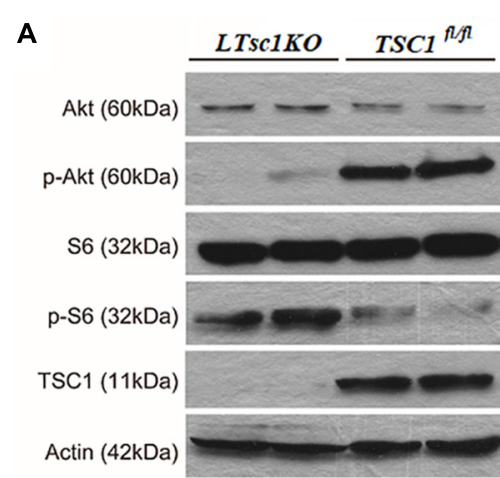

B
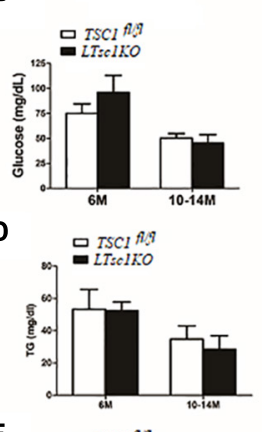

C

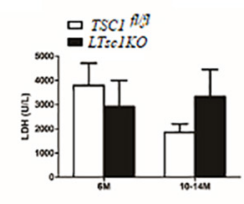

E

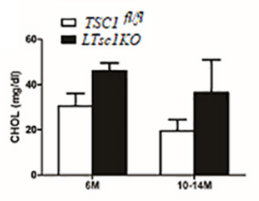

G

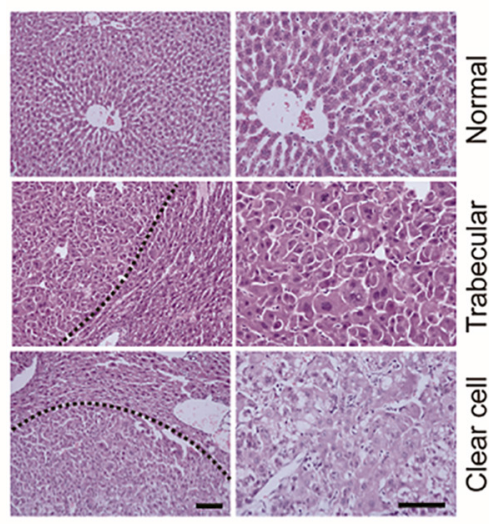

I

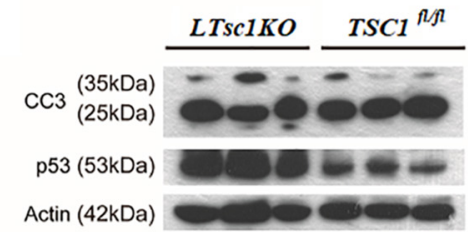

$\mathbf{F}$

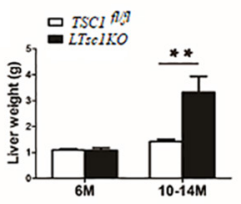

H
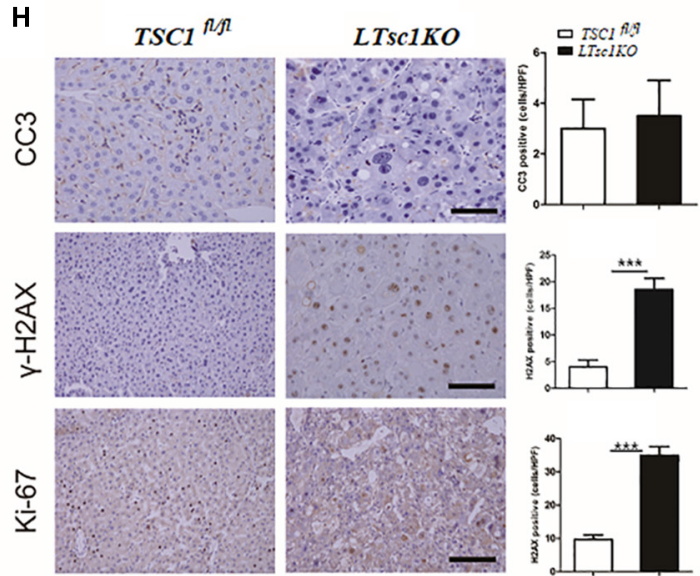

FIGURE 1 | LTsc1KO mice developed spontaneous HCC. (A) Immunoblot analysis of isolated hepatocytes from LTSC1KO and TSC1f/fl mice with TSC1, S6, Akt, and their phosphorylation antibodies to confirm activation of mTORC1. (B) Blood glucose levels in 6- and 10-14-month-old LTSC1KO and TSC1f/fl mice, respectively ( $n=45, \pm$ SEM). (C-E) Serum LDH, cholesterol, and triglyceride levels were observed in 6- and 10-14-month-old LTSC1KO and TSC ${ }^{\mathrm{fl} / \mathrm{fl}}$ mice, respectively. (F) Liver weights in 6- and 10-14-month-old LTSC1KO and TSC1 ${ }^{\mathrm{fl} / \mathrm{fl}}$ mice ( $n=45$, $\pm \mathrm{SEM}$ ), and liver weight was increased in 10-14-month-old $L T S C 1 K O$ comparing with TSC ${ }^{\mathrm{fl} / \mathrm{fl}}$ mice $\left.{ }^{* *} \mathrm{P}<0.01\right)$. (G) Liver sections showing histological types of tumor in 10-14-month-old $L T S C 1 K O$ mice. The tumor areas are marked in low magnification and high magnification to show the histological features clearly. (H) Cleaved caspase-3 (CC3), p-H2AX, and Ki67 staining of liver sections in 10-14-month-old LTSC1KO and TSC1 ${ }^{\mathrm{fl} / \mathrm{fl}}$ mice. Immunohistochemically positive cells were quantified, and results are shown in the bar graphs $(n=19, \pm$ SEM, $\left.{ }^{* * *} \mathrm{P}<0.001\right)$. (I) Hepatic CC3 and p53 protein expression in LTSC1KO and TSC1 ${ }^{\mathrm{fl} / \mathrm{fl}}$ mice.

\section{RESULTS}

\section{LTsc1KO Mice Developed Spontaneous HCC Without Systemic Glucose Tolerance}

We generated LTsc1KO mice by crossing $T S C 1^{\mathrm{fl} / \mathrm{fl}}$ with Alb-Cre mice. Deletion of the hepatic TSC1 allele was confirmed by polymerase chain reaction (PCR) of genomic DNA (Supplementary Figure S1A). Immunoblot analysis of LTsc1KO liver revealed constitutively active mTORC1 signaling in the liver, as indicated by TSC1 and the best-characterized substrates of mTORC1 S6 phosphorylation (pS6). LTsc1KO mice displayed a strong reduction in phospho-Ser473-PKB/Akt
(p-Akt). As described previously (Yecies et al., 2011), in the present study, the LTsc1KO livers display attenuation of Akt signaling due to a mTORC1-dependent negative feedback loop (Figure 1A). Surprisingly, serum parameters showed that LTsc1KO mice displayed unchanged blood glucose, triglyceride, lactate dehydrogenase $(\mathrm{LDH})$, and cholesterol levels relative to $T S C 1^{\mathrm{fl} / \mathrm{fl}}$ mice, indicating a normal insulin response in the LTsc1KO mice (Figures 1B-E). Compared with the $T S C 1^{\mathrm{fl} / \mathrm{fl}}$ mice, LTsc1KO mice did not have any decrease in body weight by 6 and $10-14$ months.

There were no detectable tumors in the LTsc1KO mice by 6 months, while 31 of 45 (68.89\%) LTsc1KO mice spontaneously developed HCC by 10-14 months. An average of three macroscopic tumors without encapsulation per liver 
was detected. Multifocal tumors were randomly distributed in all liver lobes (Supplementary Figure S1B). Consistently, these LTsc1KO mice exhibited increased liver weight by 10-14 months (Figure 1F).

Previously, tumor developed by TSC1 deficiency has been considered as benign with a high rate of apoptosis (WatayaKaneda et al., 2001). In the present study, histopathological features of HCC in LTsc1KO mice revealed large cells with enlarged and hyperchromatic nuclei and ballooning with weakly eosinophilic staining, which contained numerous microvesicular vacuoles in cytoplasm (Figure 1G). LTsc1KO did not induce significant apoptosis, as measured by cleaved caspase 3 . Phosphorylated histone-2AX ( $\gamma \mathrm{H} 2 \mathrm{AX})$-positive hepatocytes, was increased in LTsc1KO livers (Figure 1H). p53 level was increased in livers of $L T s c 1 K O$ mice. These data demonstrate that loss of TSC1 results in dramatic accumulation of p53 in response to the p53-mediated DNA damage-response pathway (Figure 1I).

\section{Hepatic Necroinflammation Was Not a Prerequisite for Spontaneous HCC in LTsc1KO Mice}

We found marked elevation of serum ALT and AST in LTsc1KO mice at 10-14 months but not at 6 months, indicating no long-term hepatic damage in LTsc1KO mice (Figure 2A). No significant changes in the number of infiltrative lymphocytes were observed in LTsc1KO mice as compared with $T S C 1^{\mathrm{fl} / \mathrm{fl}}$ mice. F4/80 staining suggested that the number of macrophages (Kupffer cells) remained unchanged in the liver from LTsc1KO mice at 6 and 10-14 months (Figure 2B).

To explore further whether there was chronic hepatic inflammation in LTsc1KO mice, we conducted a Bio-Plex mouse cytokine 23-plex assay to detect simultaneously 23 cytokines, chemokines and growth factors. It has been proved that increased IL-6 production results in activation of the oncogenic transcription factor STAT3 in liver, and TNF- $\alpha$ is a major adipose- derived cytokine and potent activator of pro-oncogenic pathways, including mTOR. The cytokine assay revealed no increased expression of inflammatory cytokines, including TNF- $\alpha$ and IL-6 (Figures 2C and Supplementary Figure S1C). Taken together, these results indicated that, the development of HCC in LTsc1KO mice was not dependent on long-term injury and necroinflammation.

We found that levels of STAT3 phosphorylation decreased in the livers of $L T s c 1 K O$ mice as compared with $T S C 1^{\mathrm{fl} / \mathrm{fl}}$ mice, and in tumors as compared with non-tumor areas of the liver in LTsc1KO mice (Figures 2D,E). It is worth noting that activity of serum and cellular GPX3 in LTsc1KO mice was similar to that in $T S C 1^{\mathrm{fl} / \mathrm{fl}}$ mice (Figures $2 \mathrm{G}, \mathbf{H}$ ). We therefore propose that HCC without necroinflammation developed possibly through inactivation of STAT3 and/or other mutations. However, we cannot completely exclude the possibility of additional mechanisms by which mTOR affects STAT3 phosphorylation.

\section{Spontaneous HCC in LTsc1KO Mice Displayed Non-cirrhotic and Chemical Carcinogenic Characteristics}

Cirrhosis is not always a prerequisite of HCC development and this might particularly apply to the HCC associated with metabolic abnormality. Sirius red staining showed that except for increased blood vessels, no obvious fibrosis was discovered, which demonstrated that hepatic mTORC1 activation did not induce liver fibrosis (Figure 2F). To investigate the molecular characteristics of HCC in LTsc1KO mice, we conducted RNA sequencing (RNA-seq) on mice at 6 and 10-14 months. The pattern of gene expression in the livers of $L T s c 1 K O$ mice was also induced in non-cirrhotic DEN-mediated HCC, including Tff3, $L y 6 d, G p c 3$, and $A f p$ (Figure 3A), and several other fetal genes that were induced in human HCC, such as Bex2, Spink1, and Rnase1. The change in Tff3 protein was confirmed by western blotting (Figure 5C).

Further analysis of the RNA-seq data revealed that the aberrant expression of enzyme genes were mainly responsible for detoxification (Figure 3B), including cytochrome P450 (CYP450), carboxylesterases (Ces), sulfotransferases (Sults), and UDP-glucuronosyltransferases (Ugts). The activities of xenobiotic metabolizing enzymes are required for activation (toxication) of important carcinogens. CYP450 enzymes are key players in the phase-I-dependent metabolism of xenobiotics. We found reduced expression of Cyp1a2, Cyp2b9, Сур2c50, Сур2c54, Сур2с67, Сур2e1, and Сур3a16, and increased expression of Cyp2b10 in the livers of LTsc1KO mice. Further gene ontology (GO) enrichment analysis revealed that differentially expressed genes were significantly enriched in biological process of chemical carcinogenesis (Figure 3C). We suggest that the first stage of carcinogenesis induced by mTORC1 activation is similar to that induced by chemical carcinogens, which results in cells with altered metabolic responsiveness and a proliferative advantage over the surrounding normal cells.

\section{LTsc1KO-Induced Spontaneous HCC Was Also Characterized by Metabolic Disorder}

We checked whether metabolic disorder occurred. Periodic acidSchiff (PAS) staining confirmed that there was less glycogen in HCC foci than adjacent hepatic tissues (Figure 4A). The metabolome was investigated in livers at 6 and 10-14 months (Figures 4B,C). Consistent with reduction of Ugtsla by RNAseq, the metabolomic changes were loss of glucuronic acid, with dramatically altered cellular metabolism at 10-14 months compared with 6 months (Figure 4D).

Pathway analysis revealed that many related metabolic pathways that influence various biological processes, such as pentose and glucuronate interconversions (carbohydrate metabolism subcategory), and the biosynthesis of ubiquinones (Figure 4E). Integrated omics profiling revealed that carcinogenesis of spontaneous HCC in LTsc1KO mice was similar to chemical carcinogenesis. Therefore, HCC induced 
A
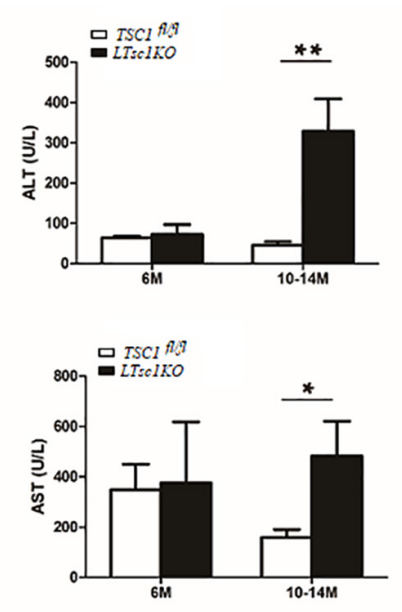

C 믐 ${ }_{\text {ISSI } A M O}$
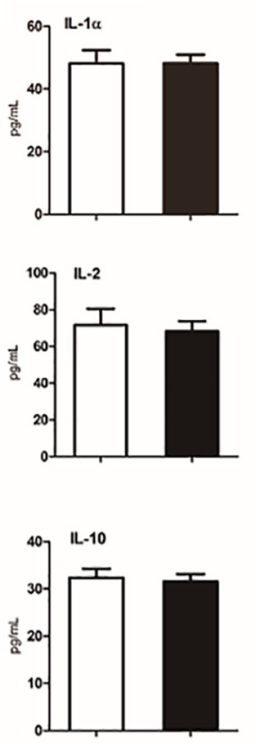

$\mathbf{F}$

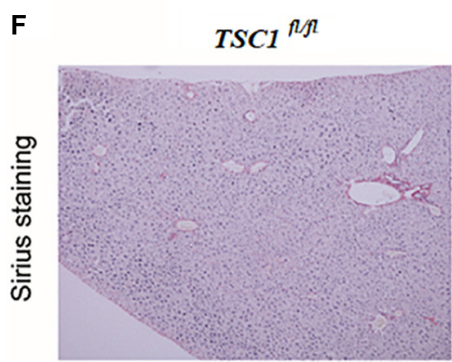

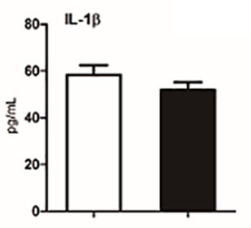
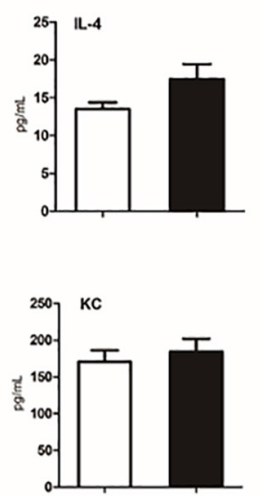

B
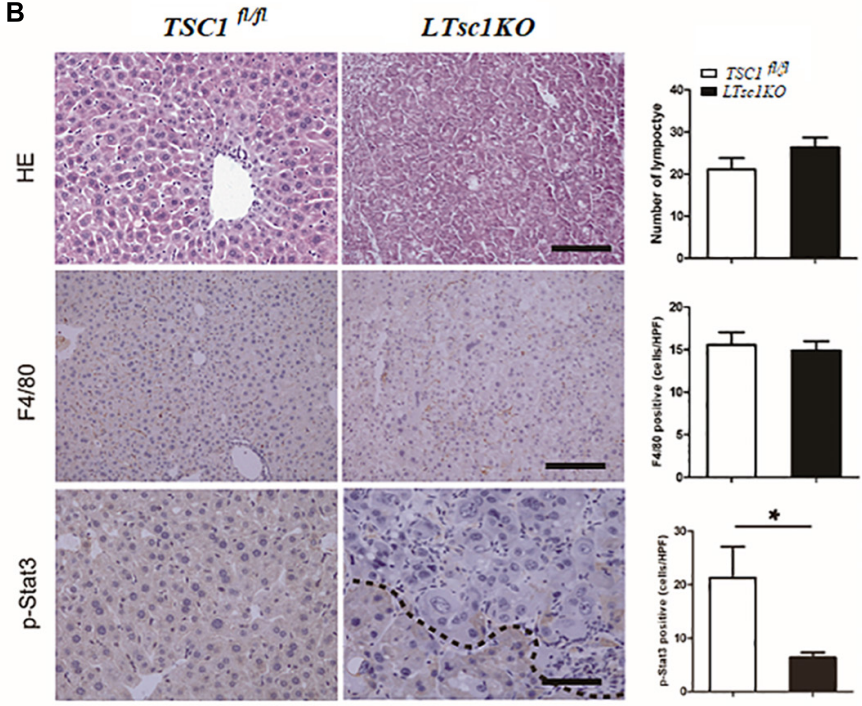

D
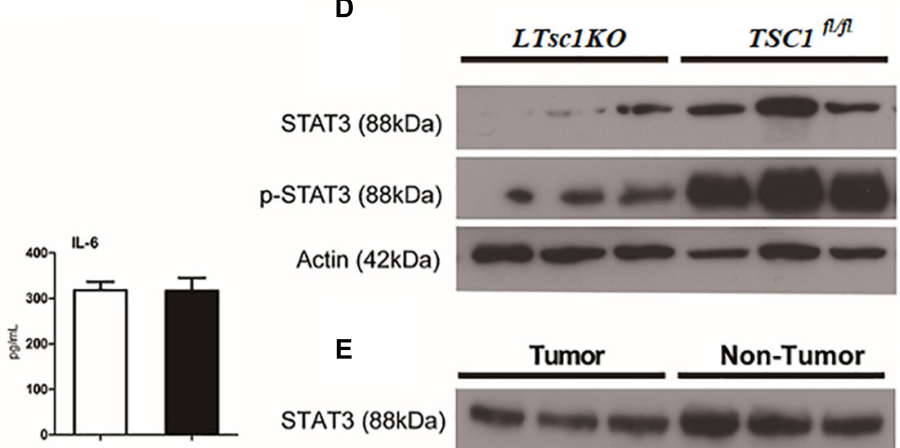

E
STAT3 $(88 \mathrm{kDa})$

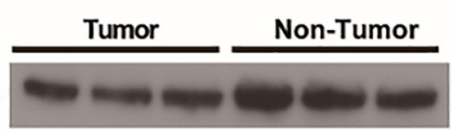

p-STAT3 (88kDa)

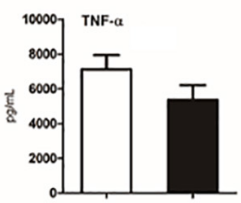

Actin $(42 \mathrm{kDa})$

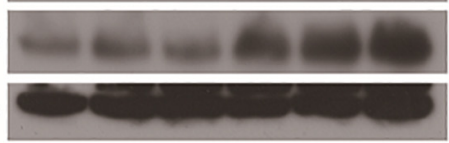

G

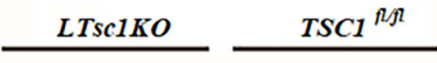

$\mathrm{Gpx3}(23 \mathrm{kDa})$

Actin (42kDa)

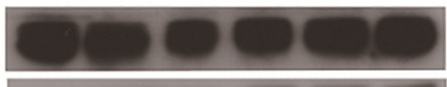

H

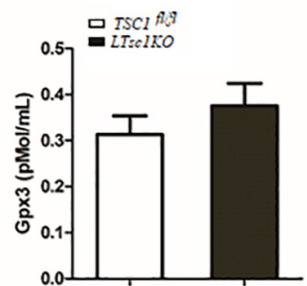

FIGURE 2 | Spontaneous HCC development in LTSC1KO mice lacked necroinflammation and fibrosis. (A) Serum ALT and AST levels in 6- and 10-14-month-old LTSC1KO and TSC $1^{\mathrm{fl} / \mathrm{fl}}$ mice $(n=29, \pm \mathrm{SEM})$. ALT and AST only increased in 10-14-month-old LTSC1KO compared with TSC1 $1^{\mathrm{fl} / \mathrm{fl}}$ mice $\left({ }^{* * P} P=0.006\right.$, and $\left.{ }^{*} P=0.046\right)$. (B) H\&E, F4/80 and p-STAT3 immunohistochemical staining of liver sections in 10-14-month-old LTSC $1 K O$ and TSC $1^{\text {fl/fl }}$ mice. Immunohistochemically positive cells were quantified, and results are shown in the bar graphs $\left(n=19, \pm\right.$ SEM, $\left.{ }^{*} P<0.05\right)$. (C) Hepatic levels of inflammatory cytokines in 10-14-month-old $L T S C 1 K O$ and TSC $1^{\mathrm{fl} / \mathrm{fl}}$ mice $\left(n=19, \pm \mathrm{SEM}\right.$ ). (D) Immunoblot analysis of hepatic STAT3 and p-STAT3 in LTSC1KO and TSC $1^{\mathrm{fl} / \mathrm{fl}}$ mice aged 10-14 months. (E) Immunoblot analysis of hepatic STAT3 and p-STAT3 in tumor and non-tumor areas of LTSC1KO mice. (F) Fibrosis was analyzed by staining liver sections with Sirius red in 10-14-month-old $L T S C 1 K O$ and TSC1//fll mice. (G,H) Serum and hepatic GPX3 level in $L T S C 1 K O$ and $T S C 1^{f / f l}$ 10-14-month-old mice $(n=14, \pm S E M)$. 


\section{A

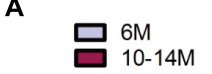

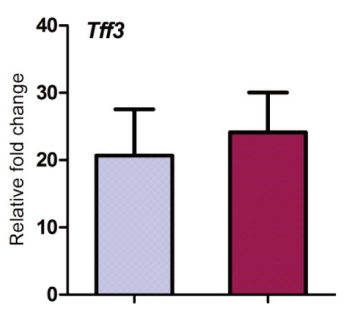
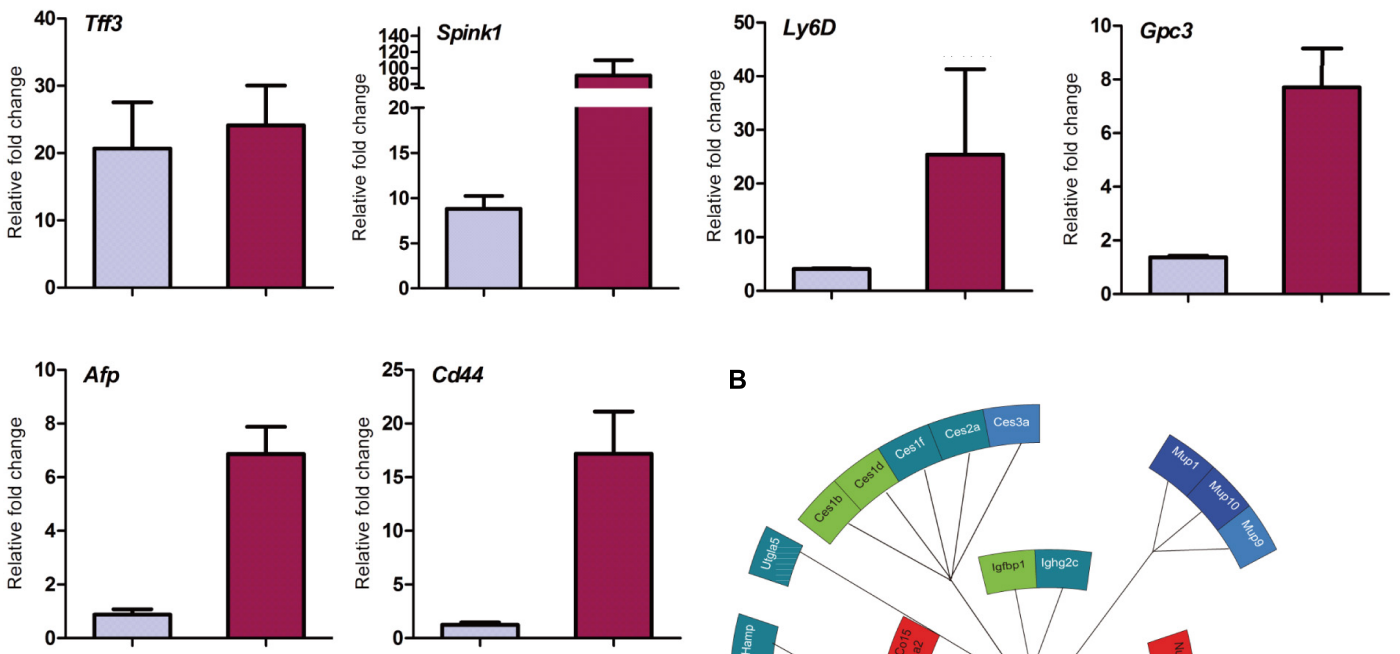

B
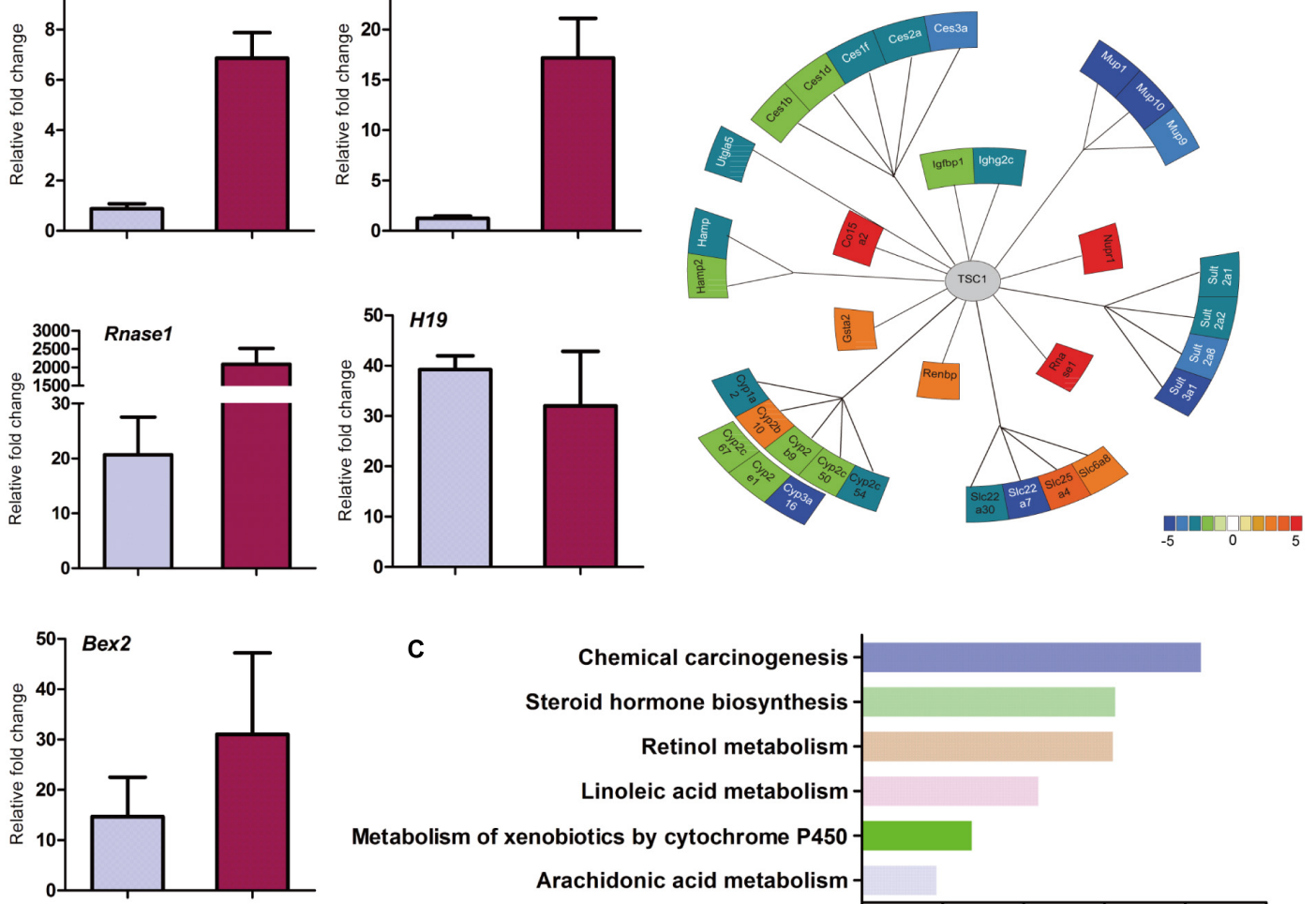

FIGURE 3 | Spontaneous HCC in LTSC1KO mice showed expression of genes commonly induced in chemical HCC. (A) qRT-PCR showing increased hepatic mRNA levels for expression of genes that are frequently induced in LTsC1KO mice aged 6 and 10-14 months, which were similar to human DEN-mediated HCC, such as Tff3, Gpc3, and Ly6d ( $n=19, \pm S E M)$. (B) Illustration of differentially expressed genes in HCC. Heat-map indicates mean values of differential expression for each gene. (C) Histographs of differentially expressed genes by pathway analysis.

by LTsc1KO was characterized by decreased glucose and TCA cycle disorder (Figure 4F). These findings suggest that specific metabolic changes, commonly seen in human hepatic carcinoma, might contribute to tumor progression in this model.

\section{Hepatocarcinogenesis Was Subsequent to Defects Upstream and Downstream of mTORC1}

RNA-seq analysis identified the dysregulated genes upstream and downstream of mTORC1, including increased mRNA level of Ragd, Ddit4, Npur1, and FGF21 (Figure 5A). Those gene changes were validated by qRT-PCR, and western blotting also revealed lower expression of Ragd, Ddit4, Npur1, and FGF21 proteins in LTsc1KO liver (Figures 5B,C), indicating that these proteins may undergo post-transcriptional modification. And no additional mutations in Ddit4, FGF21, and Nupr1 were identified (Figure 5D), indicating that protein mutations were not required for those genes after mTORC1 activation for liver carcinogenesis. While expression of Dddit4, FGF21, and Nupr1 was decreased in tumor compared to non-tumor tissue (Figure 6A). These results indicate that HCC development in LTsc1KO mice involves a complex network that requires Dddit4, FGF21, and Nupr1. Therefore, a cohort of mice aged 5 months was treated with 
A

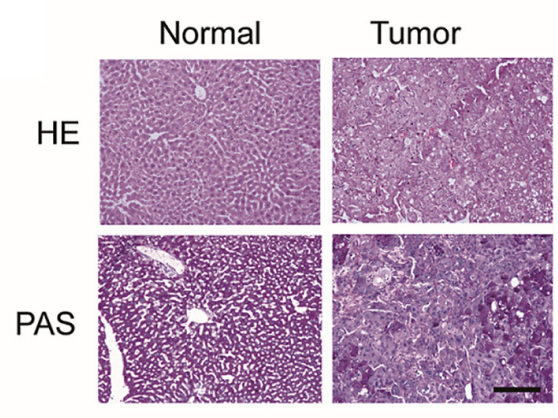

B

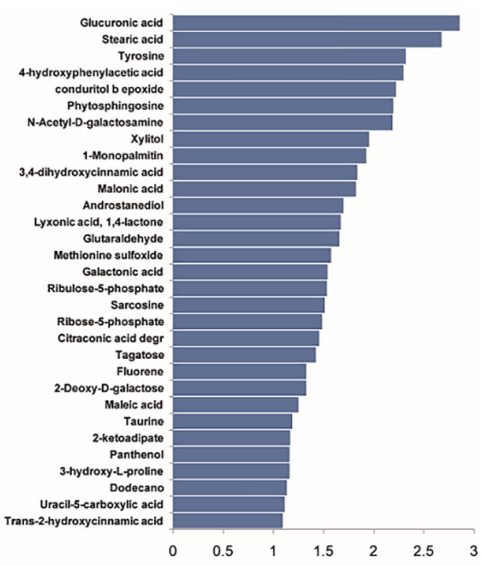

C

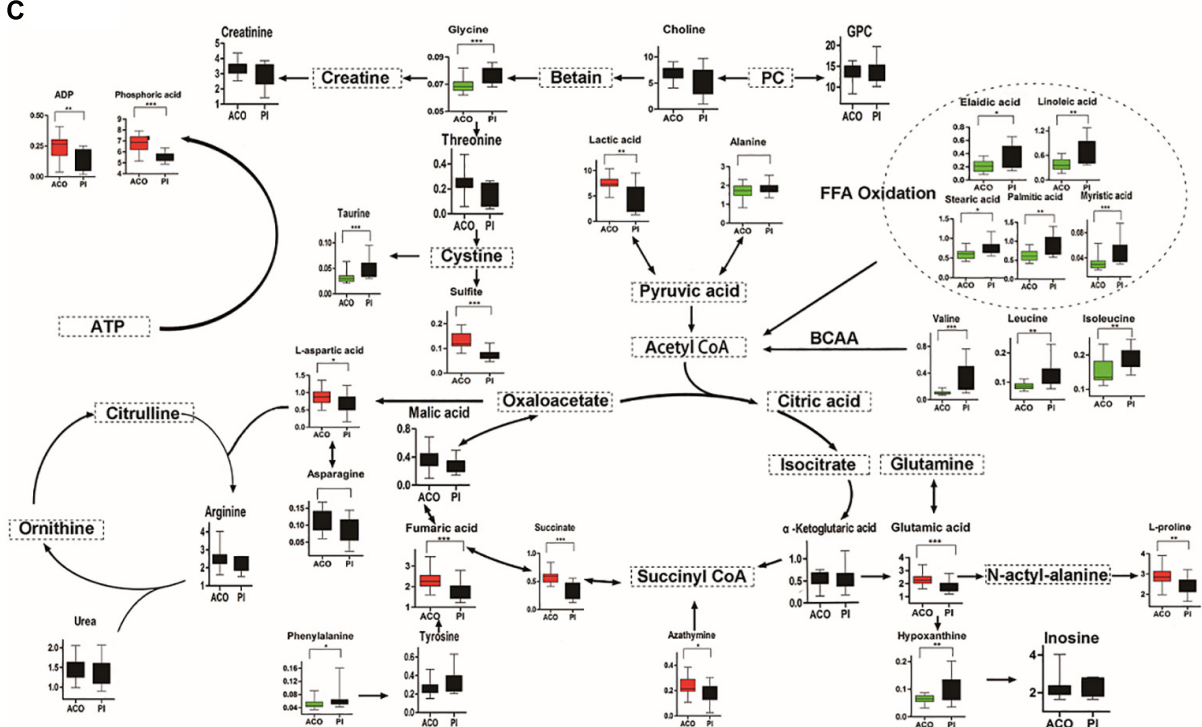

D
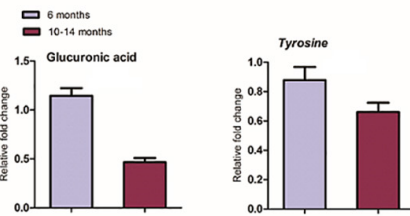

$\mathbf{F}$

E
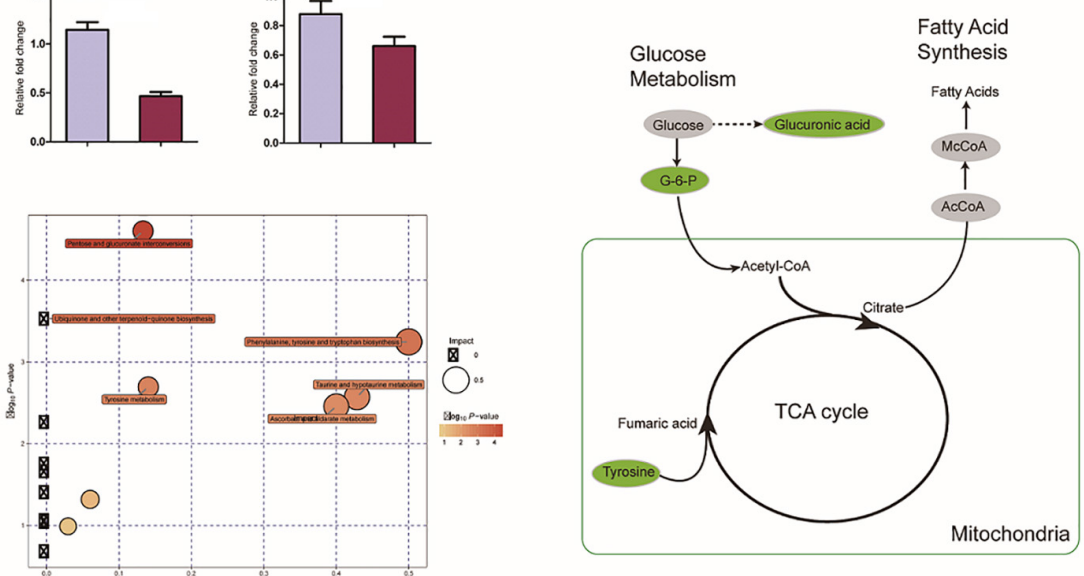

FIGURE 4 | Characterization of LTSC1KO liver-bearing carcinoma by glucuronic acid loss and metabolic disorder. (A) Liver sections were stained for glycogen with PAS. (B) Representative pictures of the significantly changed metabolites by -log10 $P$-value. (C) Metabolic pathway map in LTsc1KO mice aged 10-14 months. Green, decrease; red, increase. (D) Concentrations of hepatic glucuronic acid and tyrosine in LTSC1KO and TSC $1^{\text {fl/fl }}$ mice aged 6 and 10-14 months

$(n=19, \pm S E M)$. (E) A differential score was calculated for the KEGG pathway. Size of dots indicates the impact of the pathway and the color indicates pathways with a score of $-\log 10 P$-value. (F) Metabolic shifts in the tricarboxylic acid cycle in LTSC1KO mice aged 10-14 month. Green, decrease; gray, not measured. 
A
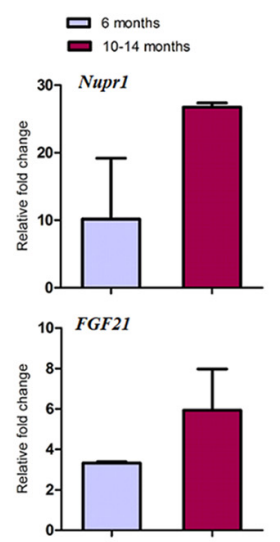
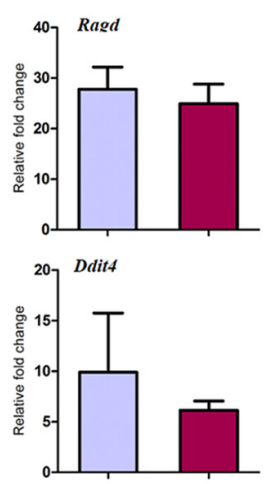

C

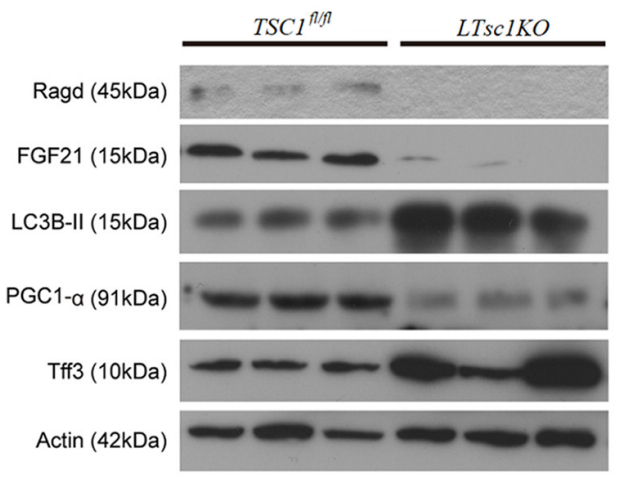

B

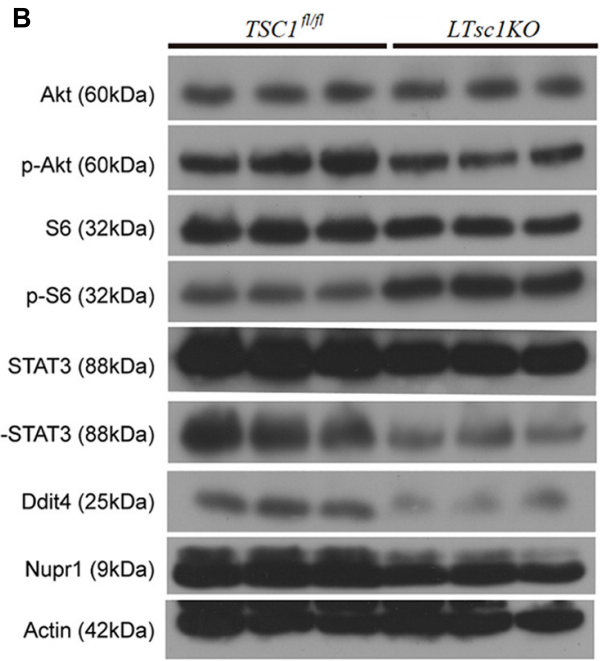

D

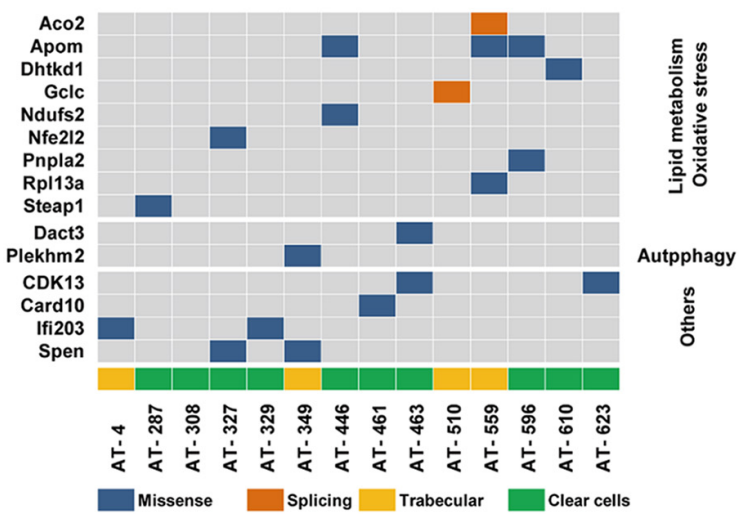

FIGURE 5 | Liver carcinogenesis subsequent to defects in mTORC1 network, and autophagy in LTsc1KO mice. (A) qRT-PCR showing hepatic Nupr1, Ragd, FGF21, and Ddit4 mRNA levels in LTSC1KO compared with TSC ${ }^{\mathrm{fl} / \mathrm{fl}}$ mice aged 6 and 10-14 months $(n=19$, \pm SEM). (B) Immunoblot analysis of hepatic Akt, p-Akt, S6, p-S6, STAT3, p-STAT3, Ddit4, and Nupr1 in LTSC1KO and TSC1 $1^{\mathrm{fl} / \mathrm{fl}}$ mice aged 10-14 months. (C) Immunoblot analysis of hepatic Ragd, FGF21, Atg5, LC3B-II, PGC1- $\alpha$, and Tff3 in LTSC1KO and TSC1 ${ }^{\mathrm{fl} / \mathrm{fl}}$ mice aged 10-14 months. (D) Representative images of mutational profile in tumor developed in $L T S C 1 K O$ mice aged 10-14 months, by RNA-seq.

rapamycin or NS three times a week for 5 months. Rapamycintreated LTsc1KO livers showed reduced LC3B-II, and increased Ddit4 and Nupr1. NS-treated LTsc1KO mice developed HCC at a rate similar to our previous cohort. Blocking mTORC1 significantly rescued the decreased expression of Nupr1 and Ddit4 in LTsc1KO liver, indicating that the upstream regulatory network dictates the pathological consequences of dysregulated mTOR signaling in liver carcinogenesis (Figure 6B). Although autophagy-related gene 5 (Atg5) was unchanged, increased levels of LC3B-II (Map1lc3b-II) were observed in LTsc1KO compared to $T S C 1^{\mathrm{fl} / \mathrm{fl}}$ mice, we also found that livers from LTsc1KO mice at 6 months age displayed accumulation of the autophagy substrate p62, which is believed to target ubiquitinated proteins and is selectively degraded by autophagy. The abundance of p62 was increased in $L T s c 1 K O$ livers (Figure 6C), indicating that mTORC1 activation by TSC1 loss caused a defect in autophagy flux. We conclude that long-term activation of mTORC1 by $L T s c 1 K O$, upstream regulators (Dddit4) of mTORC1 was impaired; meanwhile, expression of downstream genes (Nupr1 and FGF21) of mTORC1 was decreased, resulting in metabolic disorder and mitochondrial defects.

\section{Dysregulation of FGF21 Was a Later Event for Liver Carcinogenesis in LTsc1KO Mice}

There was no obvious change in expression of FGF21 in LTsc1KO mice treated with rapamycin, indicating that altered expression of FGF21 might be a critical event after long-term abrogation of Ddit4 and Nupr1 for HCC development (Figure 6B). Unchanged expression of FGF21 and decreased expression of Ddit4 and Nupr1 were confirmed in LTsc1KO mice at 6 months, indicating that FGF21 expression was an essential event during liver carcinogenesis (Figure 6C). FGF21 protein level increases in 


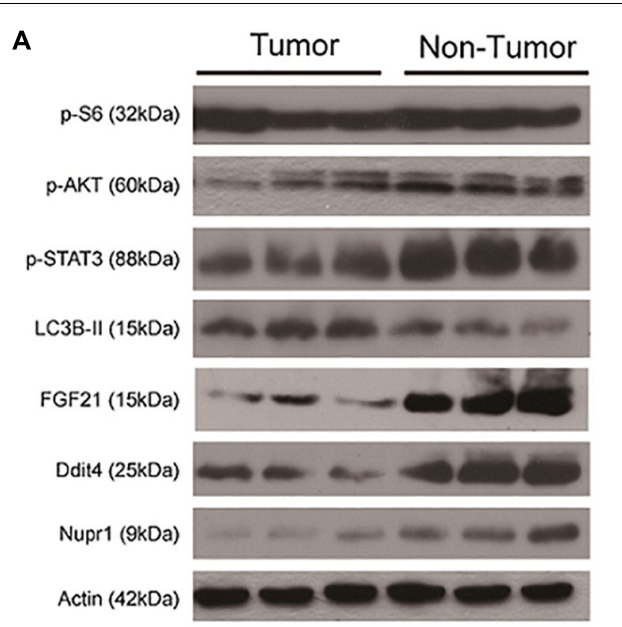

C

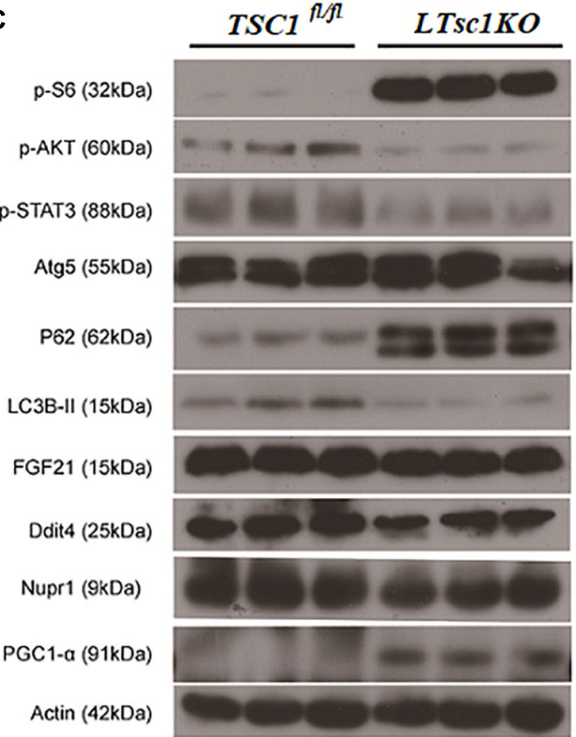

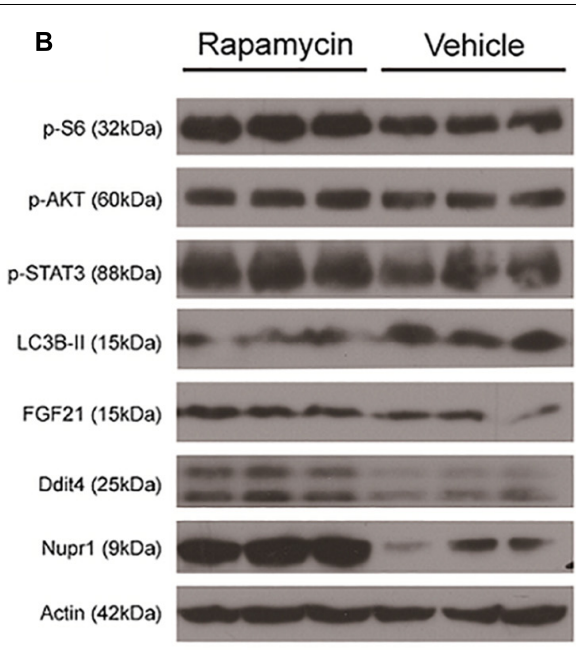

D

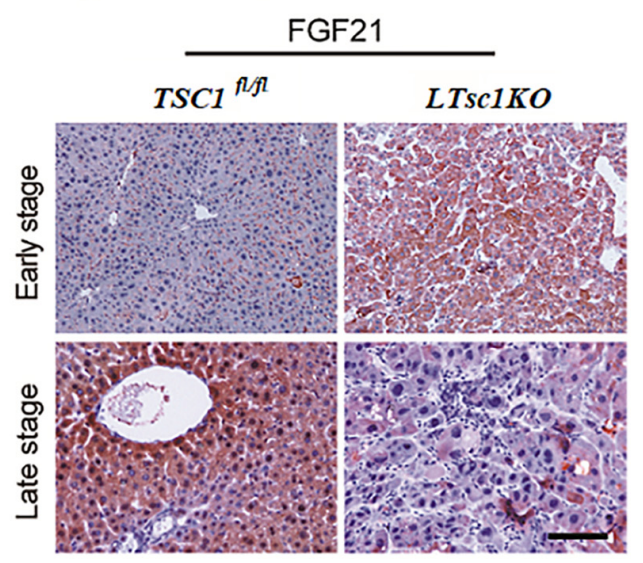

FIGURE 6 | Liver Carcinogenesis Enhanced by the mTORC1-STAT Pathway Crosstalk. (A) Immunoblot analysis of liver lysates from tumor and non-tumor areas in LTSC1KO mice aged 10-14 months. (B) Immunoblot analysis of liver lysates from LTSC1KO mice, aged 10 months, treated with vehicle or rapamycin for the preceding 5 months. (C) Immunoblot analysis of liver lysates from LTSC1KO and TSC $1^{\mathrm{fl} / \mathrm{fl}}$ mice aged 6 months. (D) FGF21 immunohistochemical staining of early and late stage liver sections from $L T S C 1 K O$ and $T S C 1^{\mathrm{fl} / \mathrm{fl}}$ mice.

liver tissues at an early stage, but decreases in liver tissues later when HCC develops (Figure 6D). Loss of FGF21 may play an important role in HCC carcinogenetic transformation during metabolic liver injury in $L T s c 1 K O$ mice. Metabolic remodeling associated with FGF21 expression also requires induction of liverintegrated stress-response-driven Nupr1 (Maida et al., 2016). In the present study, Nupr1 was decreased in LTsc1KO mice by $10-14$ months, indicating its involvement in maintaining mitochondrial defects, thereby contributing to the lipotoxic effects of liver carcinogenesis. mTORC1 negatively regulates hepatic FGF21 expression via peroxisome proliferator-activated receptor- $\gamma$ coactivator $1 \alpha$ (PGC-1 $\alpha$ ) (Estall et al., 2009). mTORC1 also controls the transcriptional activity of PGC1- $\alpha$, which induces mitochondrial biogenesis in the liver (Puigserver, 2005). At 6 months of age, mice without spontaneous HCC revealed increased PGC-1 $\alpha$ in LTsc1KO mice (Figure 6C). Conversely, at 10-14 months of age, PGC- $1 \alpha$ was markedly reduced in mice with HCC (Figure 5C). Our data also confirm that altered expression of FGF21 and Nupr1 is required during liver carcinogenesis.

\section{Crosstalk Between mTORC1 and the STAT3 Pathway Was Linked to Non-inflammatory HCC in LTsc1KO Mice}

Ddit4 is a constant modulator of p-Akt in response to growth factors and nutrients (Dennis et al., 2014), and its expression depends on activation of STAT3 (Pinno et al., 2016). In the present study, LTsc1KO mice demonstrated reduced expression of p-Akt, p-STAT3 and Ddit4 (Figure 5B). Therefore, we posit 
that Ddit4 might act as a mediator of crosstalk between mTOR and the STAT pathway. Correspondingly, lower expression of p-STAT3 were observed in LTsc1KO mice at 6 months, and LTsc1KO mice exhibited lower expression of p-STAT3 in tumor compared to non-tumor tissue. Rapamycin-treated LTsc1KO livers showed increased p-STAT3 expression compared to livers from NS-treated LTsc1KO mice. We therefore propose that the crosstalk between mTORC1 and the STAT3 pathway and/or other mutations was involved in non-inflammatory HCC development.

\section{DISCUSSION}

Given that mTORC1 regulates glucose homeostasis, lipid metabolism and cell proliferation, it is not surprising that mTOR plays a pivotal role in HCC development, and TSC1/2 mutations define a molecular subset of HCC with aggressive behavior (Ho et al., 2017). However, mechanisms of mTORC1 activationassociated HCC are not well understood. It has become necessary to understand the consequences of mTORC1 activation. To

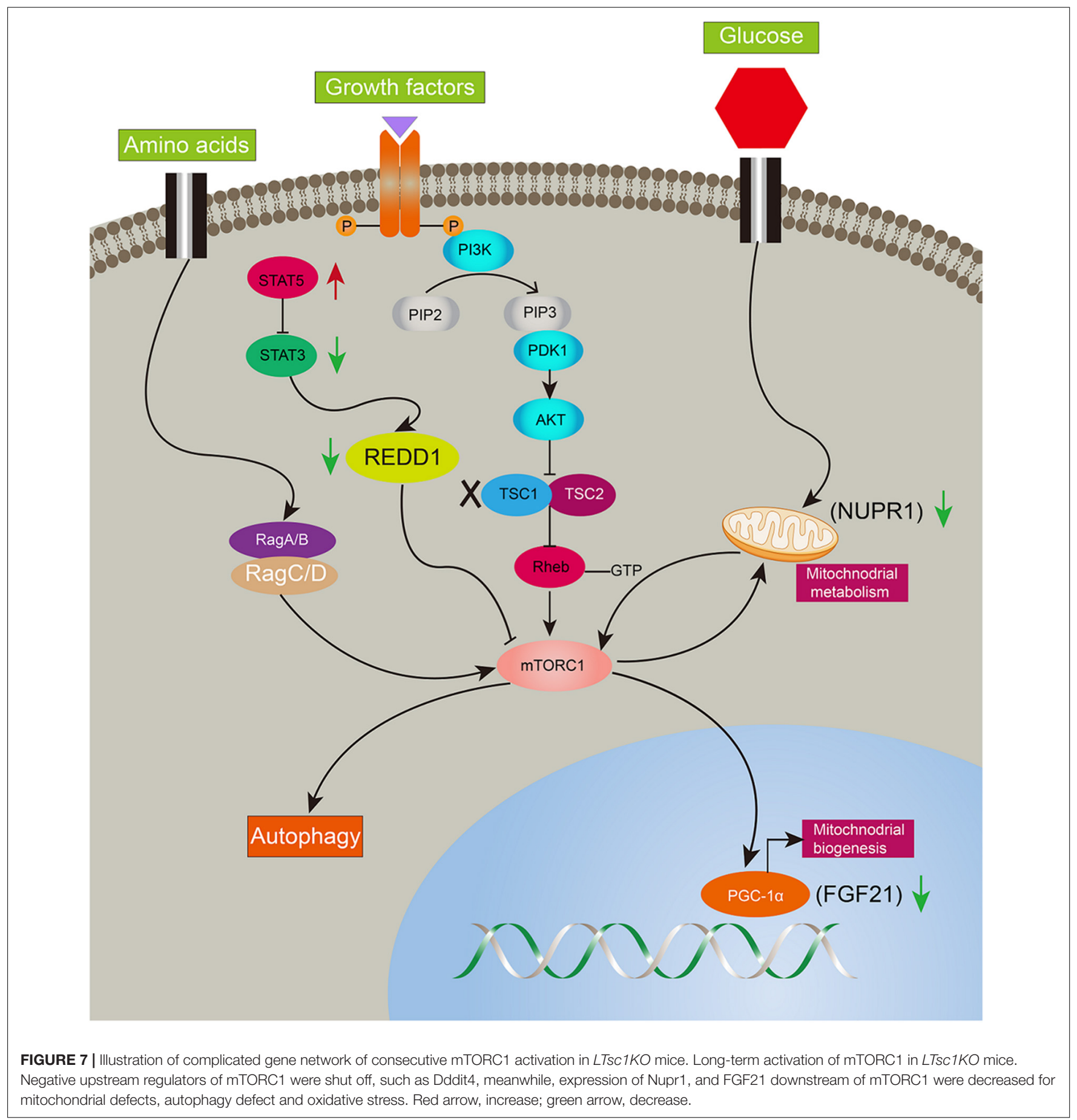


address this important issue, we aimed to provide insight into the mechanisms of HCC development in LTsc1KO mice, which is analogous to human HCC induced by NAFLD without necroinflammation and cirrhosis.

In the present study, the histopathology revealed two major types of tumor that were morphologically consistent with clear cell HCC and trabecular HCC in LTsc1KO livers. Gege expression analysis demonstrated the expression of many genes in the LTsc1KO liver that are also induced in human HCC and showed a specific molecular characteristics similar to non-cirrhotic DENmediated HCC (Degasperi and Colombo, 2016). Relationships between steatosis, steatohepatitis, cirrhosis and HCC are not necessarily linear and this pattern possibly applies to HCC arising in non-alcoholic, non-cirrhotic liver disease (Baffy et al., 2012). In NAFLD-associated HCC, there was a $23-50 \%$ prevalence of tumors developing in non-cirrhotic livers in Japan, Italy and the US (Yasui et al., 2011; Dyson et al., 2014; Mittal et al., 2015; Piscaglia et al., 2016). Therefore, in patients with NAFLD, HCC can arise in the context of non-cirrhotic liver, suggesting a specific carcinogenic pathway. Here, LTsc1KO mice provide us with an optimal model to investigate the underlying molecular mechanisms of steatohepatitic HCC development in the absence of inflammation.

HCC is often initiated by death of hepatocytes, resulting in liver injury followed by inflammation. The production of pro-tumorigenic cytokines including IL-6, which induces STAT3 activation in hepatocytes, eventually promotes compensatory proliferation in hepatocytes that have escaped cell death, and subsequently, tumor development. Hepatic deletion of genes in the PI3K-Akt-mTOR pathway, including Pten and Akt1 in Akt2, induces HCC (Horie et al., 2004; Watanabe et al., 2005; Kenerson et al., 2013; Wang et al., 2016). For example, hepatic Pten deletion results in hepatic injury and cell death, which activates tumor-initiating cells to induce HCC development (Galicia et al., 2010). Both hepatic Akt1 and Akt2 deletion mice develop HCC, which is associated with liver injury and inflammation via activated STAT3 and IL-6 expression (Wang et al., 2016). Menon et al. (2012) described the liver damage, inflammation, necrosis and regeneration in HCC developed in LTsc1KO mice.
The most intriguing and unexpected observation that was uncovered in our study was spontaneous HCC in LTsc1KO mice without long-term hepatic injury, and inflammation. The number of infiltrative lymphocytes and macrophages (Kupffer cells), and the level of cytokine remained unchanged, in the LTsc1KO mice. Moreover, decreased Akt without IL-6 elevation in $L T s c 1 K O$ mice, due to a negative regulation loop, suggested that mTORC1 activation was independent of Akt for HCC development. We revealed a previously unappreciated role for dysregulated mTORC1 signaling in promoting cancerinitiating events via inhibition of STAT3. Previous research suggested that STAT3 was activated in inflammatory HCC. It has been proved that increased IL-6 production results in activation of the oncogenic transcription factor STAT3 in liver, and TNF- $\alpha$ is a major adipose- derived cytokine and potent activator of pro-oncogenic pathways, including mTOR. Hepatocyte-specific ablation of the specific mTORC1 subunit Raptor resulted in elevated IL-6 production, activation of STAT3, and enhanced HCC development (Umemura et al., 2014). It is worth noting that mice with hepatic deletion of STAT5 are more susceptible to hepatocarcinogenesis, and the hepatic deletion of STAT5 induces STAT3 activity (Hosui et al., 2009). In the current study, we found that STAT5 is highly expressed (data not shown) and thus may inhibit the expression of STAT3. Perhaps, this is evidence to support our findings that mTORC1 might have a negative regulatory effect on STAT3.

Obesity, hepatosteatosis, insulin resistance, and chronic mTORC1 activation are associated with a pronounced increase in HCC risk (Calle et al., 2003). Our findings elucidate fundamental biochemical properties displayed by mTORC1 activation to achieve their tumor-promoting effect. There is an increasing appreciation of the fact that oncogenic signaling controls the metabolic reprogramming of cancer cells (Yecies and Manning, 2011). mTORC1, a master regulator of cellular metabolism, controls lipogenesis and glucose metabolism, however, the mechanisms and critical factors need to be elucidated. It is unclear whether the altered fatty acid composition observed in the LTsc1KO liver contributes to tumorigenesis.

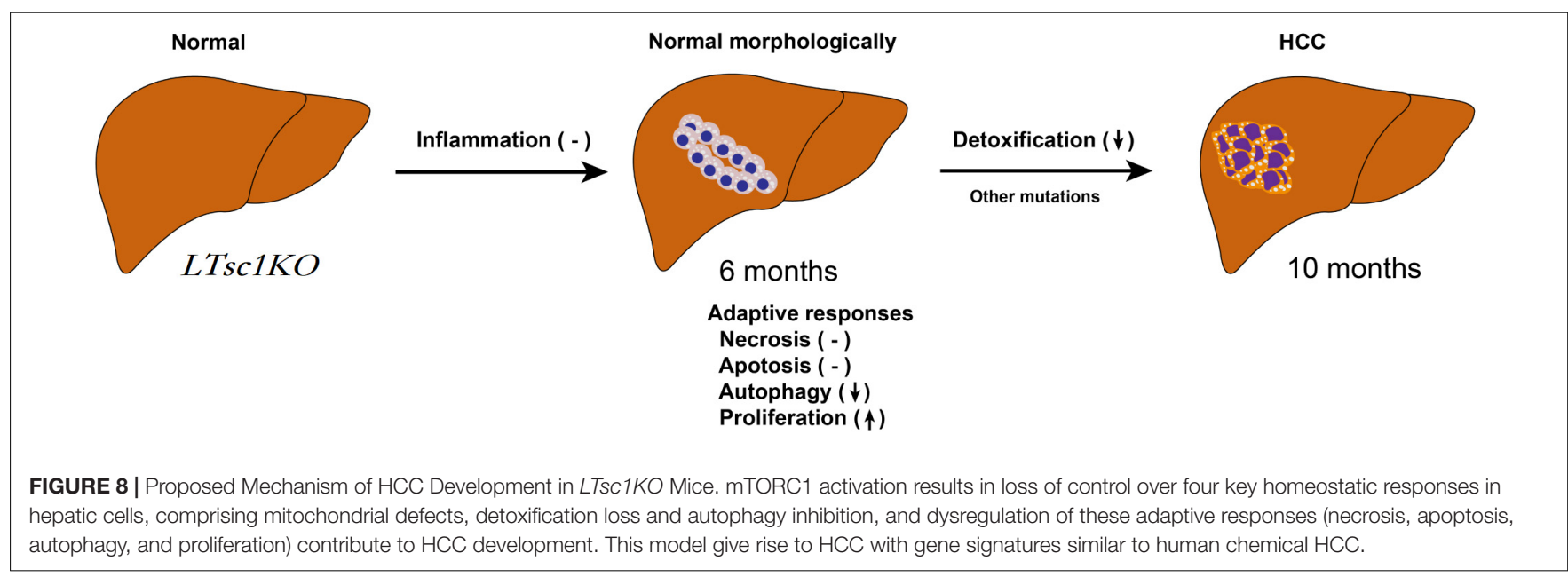


The transformation of chemicals is important in carcinogenesis the Cyp450 families are key enzymes in tumor transformation, and mediate the metabolic activation of numerous pre-carcinogens, such as Cyp1a2 and Cyp2e1, which degrade xenobiotics, steroids and fatty acids. By integrated omics profiling, we incorporated enzyme transcript levels into stearic acid accumulation. We hypothesized that metabolic disorder accompanied with inactivation of xenobiotic metabolizing enzymes, and causes defective necrosis, apoptosis and autophagy, in addition to chronic mTORC1 activation in promoting anabolic growth and proliferation.

Transformed cells survive by acquiring adaptations that allow mTORC1 to continue signaling and are insensitive to the stress of energy, oxygen and nutrient deprivation, which confers a selective growth advantage. Cancer evolves via a multistep process and molecular events in addition to TSC1 deficiency during tumor development. Kenerson et al. (2013) described that phosphorylation of InsR was reduced in TSC1deficiency tumors, which is upstream of mTORC1. A gene network containing FGF21, Ddit4, and Nupr1 could explain at least in part the transition consistent with activation of an adaptive transcriptional survival program.

Ddit4 also named Redd1, regulated in development and DNA damage responses-1, is an mTORC1 inhibitor and critical transducer of the cellular response to energy depletion (Sofer et al., 2005). Abnormalities of Ddit4-mediated signaling promote tumorigenesis (Ellisen, 2005; Pineau et al., 2010). Moreover, Ddit4 expression depends on the activation of STAT3 (Pinno et al., 2016). In the present study, Ddit4 was decreased and reversed after rapamycin given, indicating mTORC1 negative feedback on Ddit4. Nupr1, a mitochondrial defect-responsive gene, regulates autophagy induced by the lipotoxic effects of excess fatty acid accumulation in cells (Jia et al., 2016). Nupr1 has also been identified as a key regulator and metabolic switch in response to mitochondrial damage during liver cancer progression (Lee et al., 2015). In the present study, Nupr1 was decreased in LTsc1KO mice by 10-14 months, while mTORC1 negatively regulates hepatic FGF21 expression via PGC-1 $\alpha$. TSC1 deficiency abrogates FGF21-mediated inhibition of mTORC1 (Gong et al., 2016). Overexpression of hepatocyte-specific FGF21 could prevent DEN-induced liver carcinogenesis in transgenic mice (Huang et al., 2006). Mice lacking FGF21 develop substantial fatty liver and markedly exacerbated accumulation of liver triglycerides, consistent with impaired fatty acid oxidation (Fisher et al., 2014). Ddit4 is also an emerging link for crosstalk between mTOR and STAT pathways in HCC development, and highlights that consecutive STAT5 activation may partially protect $4 \mathrm{E}$ BP1 phosphorylation (Nogami et al., 2015). Insufficiency of Nupr1 in LTsc1KO mice results in non-sensitivity to a variety of stressors, including respiratory deficiency. Loss of FGF21 worsens metabolic disorder and contributes to aberrant molecular events, including lipid metabolism, in HCC development. The dysregulated expression of FGF21 is a later and critical event for HCC development. Furthermore, metabolic remodeling associated with FGF21 expression also requires induction of liver-integrated stress-response-driven Nupr1 (Maida et al.,
2016). Therefore, our data favor the possibility that LTsc1KO mice constructed a regulator network and crosstalk between mTOR and STAT3 has been attributed to oxidative, ER stress, mitochondrial dysfunction, and autophagy defects, adding a potential mechanism to induce and maintain constitutive activation of mTORC1, and mediate a metabolic feature for growth advantage (Figure 7 ).

In summary, we unraveled a comprehensive gene network and obtained mechanistic insights into how mTORC1 activation as a primary driver results in loss of control over four key homeostatic responses in hepatic cells, comprising metabolic disorder, mitochondrial defect, detoxification loss, and autophagy inhibition. mTORC1 activation, Ddit4 and STAT3 phosphorylation inhibition, indicatingdysregulation of Ddit4-mTORC1-STAT3 lop ultimately contributes to development of non-inflammatory HCC (Figure 8). The HCC that developed in LTsC1KO mice in this study provides a typical animal model for future investigation into the molecular events underlying carcinogenesis arising from non-cirrhotic liver disease.

\section{DATA AVAILABILITY STATEMENT}

All datasets generated for this study are included in the article/Supplementary Material.

\section{ETHICS STATEMENT}

All procedures involving mice was approved by the Southern Medical University Animal Care and Use Committee.

\section{AUTHOR CONTRIBUTIONS}

TL, GZ, XX, and YG designed the experiments. TL generated mice and conducted the most experiments, with assistance from LW. LW and SL made the pathological evaluations of the tissue sections. TL and GZ analyzed all the data, completed the experiments for publication, and wrote the paper. YG conceived the project, and secured funding.

\section{FUNDING}

This work was supported by the National Key R\&D Program of China (2018YFC1106400 and 018YFA0108200), Science and Technology Planning Project of Guangdong Province (2015B020229002), the National Natural Science Foundation of China (81470875, 81701580, and 81600489), the Natural Science Foundation of Guangdong Province (2014A030312013, 2018A030313128, and 2018A030313214), Guangdong key research and development plan (2019B020234003), and Science and Technology Program of Guangzhou (201604020002 and 201803010086). 


\section{SUPPLEMENTARY MATERIAL}

The Supplementary Material for this article can be found online at: https://www.frontiersin.org/articles/10.3389/fcell.2020.00225/ full\#supplementary-material

FIGURE S1 | Consequences of mTORC1 Activation by LTSC1KO. (A) Representative PCR genotyping of Alb (left) and TSC1 (right). Left: lane 1 and 3: Alb-Cre positive, lane 2 and 4: Alb-Cre negative, lane 5: water, lane 6: DNA maker 100 bp ladder. Right: lane 1: DNA double-chain TSC1-loxp site insertion, lane 2 and 4: no TSC1-loxp site insertion, lane 3: DNA single-chain TSC1-loxp site

\section{REFERENCES}

Baffy, G., Brunt, E. M., and Caldwell, S. H. (2012). Hepatocellular carcinoma in non-alcoholic fatty liver disease: an emerging menace. J. Hepatol. 56, 13841391. doi: 10.1016/j.jhep.2011.10.027

Bhat, M., Sonenberg, N., and Gores, G. J. (2013). The mTOR pathway in hepatic malignancies. Hepatology 58, 810-818. doi: 10.1002/hep.26323

Bishayee, A. (2014). The role of inflammation and liver cancer. Adv. Exp. Med. Biol. 816, 401-435. doi: 10.1007/978-3-0348-0837-8_16

Bosetti, C., Turati, F., and La Vecchia, C. (2014). Hepatocellular carcinoma epidemiology. Best Pract. Res. Clin. Gastroenterol. 28, 753-770. doi: 10.1016/ j.bpg.2014.08.007

Calle, E. E., Rodriguez, C., Walker-Thurmond, K., and Thun, M. J. (2003). Overweight, obesity, and mortality from cancer in a prospectively studied cohort of U.S. adults. N. Engl. J. Med. 348, 1625-1638. doi: 10.1056/ NEJMoa021423

Chen, X., Yamamoto, M., Fujii, K., Nagahama, Y., Ooshio, T., Xin, B., et al. (2015). Differential reactivation of fetal/neonatal genes in mouse liver tumors induced in cirrhotic and non-cirrhotic conditions. Cancer Sci. 106, 972-981. doi: $10.1111 /$ cas. 12700

Degasperi, E., and Colombo, M. (2016). Distinctive features of hepatocellular carcinoma in non-alcoholic fatty liver disease. Lancet Gastroenterol. Hepatol. 1, 156-164. doi: 10.1016/S2468-1253(16)30018-8

Dennis, M. D., Coleman, C. S., Berg, A., Jefferson, L. S., and Kimball, S. R. (2014). REDD1 enhances protein phosphatase 2A-mediated dephosphorylation of Akt to repress mTORC1 signaling. Sci. Signal. 7:ra68. doi: 10.1126/scisignal. 2005103

Dyson, J., Jaques, B., Chattopadyhay, D., Lochan, R., Graham, J., Das, D., et al. (2014). Hepatocellular cancer: the impact of obesity, type 2 diabetes and a multidisciplinary team. J. Hepatol. 60, 110-117. doi: 10.1016/j.jhep.2013.08.011

Ellisen, L. W. (2005). Growth control under stress: mTOR regulation through the REDD1-TSC pathway. Cell Cycle 4, 1500-1502. doi: 10.4161/cc.4.11.2139

Estall, J. L., Ruas, J. L., Choi, C. S., Laznik, D., Badman, M., Maratos-Flier, E., et al. (2009). PGC-1alpha negatively regulates hepatic FGF21 expression by modulating the heme/Rev-Erb(alpha) axis. Proc. Natl. Acad. Sci. U.S.A. 106, 22510-22515. doi: 10.1073/pnas.0912533106

Fisher, F. M., Chui, P. C., Nasser, I. A., Popov, Y., Cunniff, J. C., Lundasen, T., et al. (2014). Fibroblast growth factor 21 limits lipotoxicity by promoting hepatic fatty acid activation in mice on methionine and choline-deficient diets. Gastroenterology 147, 1073-1083.e6. doi: 10.1053/j.gastro.2014.07.044

Galicia, V. A., He, L., Dang, H., Kanel, G., Vendryes, C., French, B. A., et al. (2010). Expansion of hepatic tumor progenitor cells in Pten-null mice requires liver injury and is reversed by loss of AKT2. Gastroenterology 139, 2170-2182. doi: 10.1053/j.gastro.2010.09.002

Gong, Q., Hu, Z., Zhang, F., Cui, A., Chen, X., Jiang, H., et al. (2016). Fibroblast growth factor 21 improves hepatic insulin sensitivity by inhibiting mammalian target of rapamycin complex 1 in mice. Hepatology 64, 425-438. doi: 10.1002/ hep. 28523

Guertin, D. A., and Sabatini, D. M. (2007). Defining the role of mTOR in cancer. Cancer Cell 12, 9-22. doi: 10.1016/j.ccr.2007.05.008

Guzman, G., Brunt, E. M., Petrovic, L. M., Chejfec, G., Layden, T. J., and Cotler, S. J. (2008). Does nonalcoholic fatty liver disease predispose patients to hepatocellular carcinoma in the absence of cirrhosis? Arch. Pathol. Lab. Med. 132, 1761-1766. doi: 10.1043/1543-2165-132.11.1761 insertion, lane 5: water, lane 6: DNA maker 100 bp ladder. Alb-Cre recombinase is a site-specific enzyme, could catalyze recombination between two TSC1-Ioxp sites, delation the TSC1 gene. Take together, lane 1 is Alb-TSC $1^{\text {t/fll }}$ mice, also refers to $L T S C 1 K O$ mice, lane 2 and 4 is TSC1 +/+ mice, also refers to C57BL/6j mice, lane 3 is $A / b-T S C 1^{\mathrm{fl} /+}$ mice. (B) (Related to Figure 1). Representative pictures of livers in 10-14 months old $L T S C 1 K O$ and $T S C 1^{f / f f l}$ mice. (C) (Related to Figure 2). Hepatic levels of inflammatory cytokines quantified in 10-14-month-old LTSC1KO and TSC $1^{\mathrm{fl} / \mathrm{fl}}$ mice, including IL-3, IL-5, IL-12(P40), IL-12(P70), macrophage inflammatory protein (MIP) $1 \alpha$, MIP $1 \beta$, interferon- $\gamma$, and monocyte chemotactic protein 1 ( $n=19, \pm$ SEM).

TABLE S1 | Primer sequences used for RT-PCR.

Ho, D. W. H., Chan, L. K., Chiu, Y. T., Xu, I. M. J., Poon, R. T. P., Cheung, T. T., et al. (2017). TSC1/2 mutations define a molecular subset of HCC with aggressive behaviour and treatment implication. Gut 66, 1496-1506. doi: 10. 1136/gutjnl-2016-312734

Horie, Y., Suzuki, A., Kataoka, E., Sasaki, T., Hamada, K., Sasaki, J., et al. (2004). Hepatocyte-specific Pten deficiency results in steatohepatitis and hepatocellular carcinomas. J. Clin. Invest. 113, 1774-1783. doi: 10.1172/JCI2 0513

Hosui, A., Kimura, A., Yamaji, D., Zhu, B. M., Na, R., and Hennighausen, L. (2009). Loss of STAT5 causes liver fibrosis and cancer development through increased TGF- $\{$ beta\} and STAT3 activation. J. Exp. Med. 206, 819-831. doi: $10.1084 /$ jem. 20080003

Huang, X., Yu, C., Jin, C., Yang, C., Xie, R., Cao, D., et al. (2006). Forced expression of hepatocyte-specific fibroblast growth factor 21 delays initiation of chemically induced hepatocarcinogenesis. Mol. Carcinog. 45, 934-942. doi: 10.1002/mc. 20241

Inarrairaegui, M., Melero, I., and Sangro, B. (2018). Immunotherapy of hepatocellular carcinoma: facts and hopes. Clin. Cancer Res. 24, 1518-1524. doi: 10.1158/1078-0432.CCR-17-0289

Jia, S. N., Lin, C., Chen, D. F., Li, A. Q., Dai, L., Zhang, L., et al. (2016). The transcription factor $\mathrm{p} 8$ regulates autophagy in response to palmitic acid stress via a mammalian target of rapamycin (mTOR)-independent signaling pathway. J. Biol. Chem. 291, 4462-4472. doi: 10.1074/jbc.M115.675793

Kenerson, H. L., Yeh, M. M., Kazami, M., Jiang, X., Riehle, K. J., McIntyre, R. L., et al. (2013). Akt and mTORC1 have different roles during liver tumorigenesis in mice. Gastroenterology 144, 1055-1065. doi: 10.1053/j.gastro.2013.01.053

Kwiatkowski, D. J., Zhang, H., Bandura, J. L., Heiberger, K. M., Glogauer, M., ElHashemite, N., et al. (2002). A mouse model of TSC1 reveals sex-dependent lethality from liver hemangiomas, and up-regulation of p70S6 kinase activity in Tsc1 null cells. Hum. Mol. Genet. 11, 525-534.

Laplante, M., and Sabatini, D. M. (2009). mTOR signaling at a glance. J. Cell Sci. 122(Pt 20), 3589-3594. doi: 10.1242/jcs.051011

Lee, D. F., Kuo, H. P., Chen, C. T., Wei, Y., Chou, C. K., Hung, J. Y., et al. (2008). IKKbeta suppression of TSC1 function links the mTOR pathway with insulin resistance. Int. J. Mol. Med. 22, 633-638.

Lee, Y. K., Jee, B. A., Kwon, S. M., Yoon, Y. S., Xu, W. G., Wang, H. J., et al. (2015). Identification of a mitochondrial defect gene signature reveals NUPR1 as a key regulator of liver cancer progression. Hepatology 62, 1174-1189. doi: 10.1002/hep.27976

Maida, A., Zota, A., Sjoberg, K. A., Schumacher, J., Sijmonsma, T. P., Pfenninger, A., et al. (2016). A liver stress-endocrine nexus promotes metabolic integrity during dietary protein dilution. J. Clin. Invest. 126, 3263-3278. doi: 10.1172/ JCI85946

Menon, S., Yecies, J. L., Zhang, H. H., Howell, J. J., Nicholatos, J., Harputlugil, E., et al. (2012). Chronic activation of mTOR complex 1 is sufficient to cause hepatocellular carcinoma in mice. Sci. Signal. 5:ra24. doi: 10.1126/scisignal. 2002739

Mittal, S., and El-Serag, H. B. (2013). Epidemiology of hepatocellular carcinoma: consider the population. J. Clin. Gastroenterol. 47(Suppl.), S2-S6. doi: 10.1097/ MCG.0b013e3182872f29

Mittal, S., Sada, Y. H., El-Serag, H. B., Kanwal, F., Duan, Z., Temple, S., et al. (2015). Temporal trends of nonalcoholic fatty liver disease-related hepatocellular carcinoma in the veteran affairs population. Clin. Gastroenterol. Hepatol. 13, 594-601. doi: 10.1016/j.cgh.2014.08.013 
Mohamad, B., Shah, V., Onyshchenko, M., Elshamy, M., Aucejo, F., Lopez, R., et al. (2016). Characterization of hepatocellular carcinoma (HCC) in nonalcoholic fatty liver disease (NAFLD) patients without cirrhosis. Hepatol. Int. 10, 632-639. doi: 10.1007/s12072-015-9679-0

Moriya, K., Nakagawa, K., Santa, T., Shintani, Y., Fujie, H., Miyoshi, H., et al. (2001). Oxidative stress in the absence of inflammation in a mouse model for hepatitis C virus-associated hepatocarcinogenesis. Cancer Res. 61, 4365-4370.

Nogami, A., Oshikawa, G., Okada, K., Fukutake, S., Umezawa, Y., Nagao, T., et al. (2015). FLT3-ITD confers resistance to the PI3K/Akt pathway inhibitors by protecting the mTOR/4EBP1/Mcl-1 pathway through STAT5 activation in acute myeloid leukemia. Oncotarget 6, 9189-9205. doi: 10.18632/oncotarget.3279

Pineau, P., Volinia, S., McJunkin, K., Marchio, A., Battiston, C., Terris, B., et al. (2010). miR-221 overexpression contributes to liver tumorigenesis. Proc. Natl. Acad. Sci. U.S.A. 107, 264-269. doi: 10.1073/pnas.0907904107

Pinno, J., Bongartz, H., Klepsch, O., Wundrack, N., Poli, V., Schaper, F., et al. (2016). Interleukin-6 influences stress-signalling by reducing the expression of the mTOR-inhibitor REDD1 in a STAT3-dependent manner. Cell. Signal. 28, 907-916. doi: 10.1016/j.cellsig.2016.04.004

Piscaglia, F., Svegliati-Baroni, G., Barchetti, A., Pecorelli, A., Marinelli, S., Tiribelli, C., et al. (2016). Clinical patterns of hepatocellular carcinoma in nonalcoholic fatty liver disease: a multicenter prospective study. Hepatology 63, 827-838. doi: $10.1002 /$ hep. 28368

Puigserver, P. (2005). Tissue-specific regulation of metabolic pathways through the transcriptional coactivator PGC1-alpha. Int. J. Obes. 29(Suppl. 1), S5-S9. doi: $10.1038 /$ sj.ijo.0802905

Sofer, A., Lei, K., Johannessen, C. M., and Ellisen, L. W. (2005). Regulation of mTOR and cell growth in response to energy stress by REDD1. Mol. Cell. Biol. 25, 5834-5845. doi: 10.1128/MCB.25.14.5834-5845.2005

Totoki, Y., Tatsuno, K., Yamamoto, S., Arai, Y., Hosoda, F., Ishikawa, S., et al. (2011). High-resolution characterization of a hepatocellular carcinoma genome. Nat. Genet. 43, 464-469. doi: 10.1038/ng.804

Umemura, A., Park, E. J., Taniguchi, K., Lee, J. H., Shalapour, S., Valasek, M. A., et al. (2014). Liver damage, inflammation, and enhanced tumorigenesis after persistent mTORC1 inhibition. Cell Metab. 20, 133-144. doi: 10.1016/j.cmet. 2014.05.001

Wang, Q., Yu, W. N., Chen, X., Peng, X. D., Jeon, S. M., Birnbaum, M. J., et al. (2016). Spontaneous hepatocellular carcinoma after the combined deletion of Akt isoforms. Cancer Cell 29, 523-535. doi: 10.1016/j.ccell.2016. 02.008

Watanabe, S., Horie, Y., and Suzuki, A. (2005). Hepatocyte-specific Pten-deficient mice as a novel model for nonalcoholic steatohepatitis and hepatocellular carcinoma. Hepatol. Res. 33, 161-166. doi: 10.1016/j.hepres.2005.09.026

Wataya-Kaneda, M., Kaneda, Y., Hino, O., Adachi, H., Hirayama, Y., Seyama, K., et al. (2001). Cells derived from tuberous sclerosis show a prolonged $\mathrm{S}$ phase of the cell cycle and increased apoptosis. Arch. Dermatol. Res. 293, 460-469. doi: 10.1007/s004030100259

Yasui, K., Hashimoto, E., Komorizono, Y., Koike, K., Arii, S., Imai, Y., et al. (2011). Characteristics of patients with nonalcoholic steatohepatitis who develop hepatocellular carcinoma. Clin. Gastroenterol. Hepatol. 9, 428-433. doi: 10. 1016/j.cgh.2011.01.023

Yecies, J. L., and Manning, B. D. (2011). Transcriptional control of cellular metabolism by mTOR signaling. Cancer Res. 71, 2815-2820. doi: 10.1158/00085472.CAN-10-4158

Yecies, J. L., Zhang, H. H., Menon, S., Liu, S., Yecies, D., Lipovsky, A. I., et al. (2011). Akt stimulates hepatic SREBP1c and lipogenesis through parallel mTORC1dependent and independent pathways. Cell Metab. 14, 21-32. doi: 10.1016/j. cmet.2011.06.002

Yen, C. J., Lin, Y. J., Yen, C. S., Tsai, H. W., Tsai, T. F., Chang, K. Y., et al. (2012). Hepatitis B virus $\mathrm{X}$ protein upregulates mTOR signaling through IKKbeta to increase cell proliferation and VEGF production in hepatocellular carcinoma. PLoS One 7:e41931. doi: 10.1371/journal.pone.0041931

Yu, M. C., and Yuan, J. M. (2004). Environmental factors and risk for hepatocellular carcinoma. Gastroenterology 127(5 Suppl. 1), S72-S78.

Conflict of Interest: The authors declare that the research was conducted in the absence of any commercial or financial relationships that could be construed as a potential conflict of interest.

Copyright (c) 2020 Li, Zhang, Wang, Li, Xu and Gao. This is an open-access article distributed under the terms of the Creative Commons Attribution License (CC BY). The use, distribution or reproduction in other forums is permitted, provided the original author(s) and the copyright owner(s) are credited and that the original publication in this journal is cited, in accordance with accepted academic practice. No use, distribution or reproduction is permitted which does not comply with these terms. 